\title{
Ovarian Abscess Caused by Peptostreptococcus magnus Following Transvaginal Ultrasound-Guided Aspiration of Ovarian Endometrioma and Fixation With Pure Ethanol
}

\author{
H. Mikamo,* K. Kawazoe, Y. Sato, M. Itoh, and T. Tamaya \\ Department of Obstetrics and Gynecology, School of Medicine, Gifu University, Gifu-city, Gifu, Japan
}

\begin{abstract}
Background: Transvaginal ultrasound-guided aspiration of ovarian endometrioma has been applied and emphasized as a safe and simple procedure.

Case: Two 27-year-old infertile women, both gravida 0, para 0, underwent medical follow-up examinations for cases of ovarian endometrioma. Both had undergone transvaginal ultrasoundguided aspiration of ovarian endometrioma. Because both were continuously febrile and had abdominal pain and cysts with tenderness in spite of antibiotic therapies, both underwent laparotomies for treatment. In both cases, enucleation of the ovarian abscess revealed purulent and malodorous fluid that demonstrated Peptostreptococcus magnus in culture.

Conclusion: We theorize that following transvaginal ultrasound-guided aspiration of ovarian endometrioma and fixation with pure ethanol, anaerobic infection by $P$. magnus occurred, and a cyst formed in the abscess. Infect. Dis. Obstet. Gynecol. 6:66-68, 1998. ๑1998 Wiley-Liss, Inc.
\end{abstract}

$P$ elvic endometriosis usually coincides with ovarian endometrioma, or so-called chocolate cysts, that is often resistant to conservative drug therapy. The control of the growth of the endometrioma has been proposed medically, ${ }^{1}$ surgically, and even laparoscopically. ${ }^{2}$ In addition, transvaginal ultrasound-guided aspiration of ovarian endometrioma has been applied ${ }^{3-5}$ and emphasized as a safe and simple procedure. ${ }^{4-6}$ Pure ethanol can fix the tissues, which could be clinically applicable for the treatment of the endometrioma wall.

We report two cases of abscess in an ovarian endometrioma infected with the anaerobe Peptostreptococcus magnus following transvaginal aspiration and fixation with ethanol.

\section{CASE REPORTS}

\section{Case I}

A 27-year-old infertile woman, gravida 0 , para 0 , underwent medical follow-up examination for her pelvic endometriosis in Gifu University Hospital. Ultrasonographic examination indicated the presence of a $6.9 \times 5.8-\mathrm{cm}$ ovarian cyst that was uncontrollable. Serum levels of CA125 and CA19-9 were $68.0 \mathrm{IU} / \mathrm{ml}$ and $64.5 \mathrm{IU} / \mathrm{ml}$, respectively. We decided on the following procedure to treat the cyst. Informed consent for the procedure was obtained from the patient. Under venous anesthesia and transvaginal ultrasound guidance, a 16-gauge needle was inserted in the right ovarian endometrioma, $30 \mathrm{ml}$ of tarry fluid aspirated, and $10 \mathrm{ml}$ of 99\% ethanol instilled to decrease the activity of the

*Correspondence to: Dr. Hiroshige Mikamo, Department of Obstetrics and Gynecology, Gifu University School of Medicine, 40, Tsukasa-machi, Gifu city, Gifu 500-8705, Japan. 


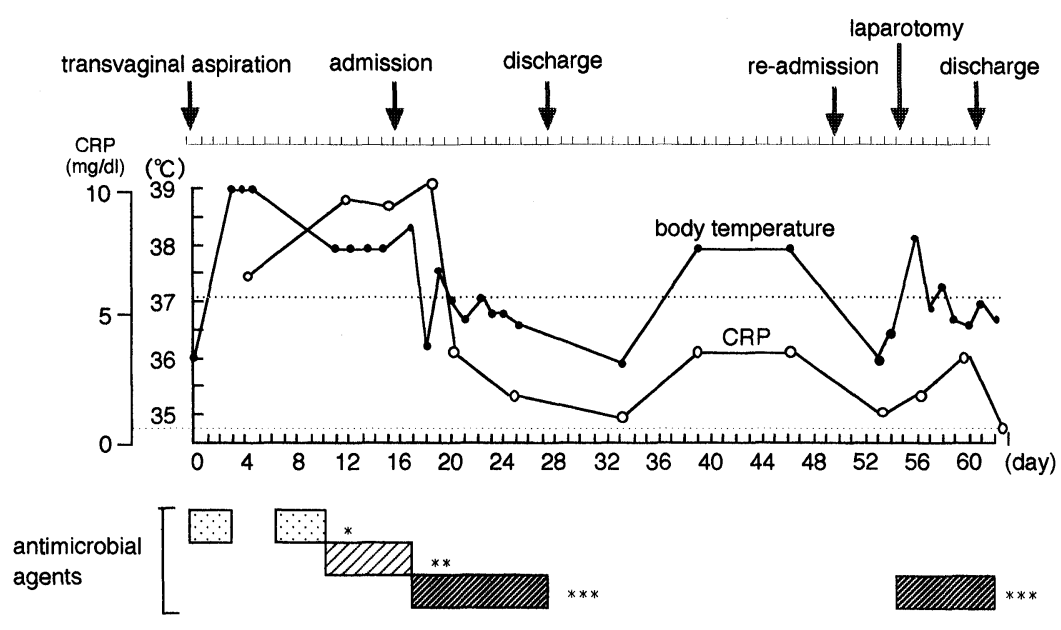

Fig. I. Clinical course of case I. * = Cefteram pivoxil, $300 \mathrm{mg} /$ day orally, three times a day. $* *=$ Cefminox, $2.0 \mathrm{~g} /$ day intravenously, two times per day. ${ }^{* * *}=$ Panipenem/betamipron, I.0 g/day intravenously, two times per day.

ovarian endometrioma by damaging the endometriosis tissue. After 10 minutes of ethanol exposure, whole tarry ethanol was removed by aspiration. The size of the ovarian endometrioma decreased to $1.7 \times 2.6 \mathrm{~cm}$. Cefteram pivoxil, $300 \mathrm{mg} /$ day orally, three times a day for three days, was given to prevent postoperative infection. The patient was monitored in the outpatient clinic. On the 4th day posttreatment, the patient became febrile. On the 6th day posttreatment, she visited our outpatient clinic again, and cefteram pivoxil, $300 \mathrm{mg} /$ day orally, three times a day for four days, was prescribed. On the 10th day posttreatment, a right cyst of $7.7 \times 2.6 \mathrm{~cm}$ with tenderness was detected. Therefore, cefminox, $2.0 \mathrm{~g} / \mathrm{day}$ intravenously, two times per day for six days, was administered. Because the patient's lower abdominal pain increased in severity on the 16th postoperative day, she was admitted to our hospital and panipenem/ betamipron, $1.0 \mathrm{~g} /$ day intravenously, two times per day for 12 days, was administered. Beginning on the 17 th postoperative day, the patient became afebrile, and she was later discharged. Because febrile episodes, abdominal pains, and cyst size had recurred throughout the clinical course and an ovarian abscess formed, laparotomy was chosen for treatment. Enucleation of the right ovarian abscess revealed purulent and malodorous fluid that demonstrated P. magnus in culture, as well as on the results of a gram stain. Afterwards, the patient received panipenem/betamipron, $1.0 \mathrm{~g} /$ day for seven days, and her recovery was uneventful (Fig. 1).

\section{Case 2}

A 27-year-old single woman, gravida 0 , para 0 , reported mild dysmenorrhea and was referred to Gifu University Hospital due to the possibility of pelvic endometriosis. Ultrasonographic examination suggested a $5.0 \times 5.0-\mathrm{cm}$ ovarian cyst, which was consistent with ovarian endometriosis. Serum levels of CA125 and CA19-9 were 71.0 IU/ml and 44.5 IU/ $\mathrm{ml}$, respectively. Informed consent was obtained from the patient, and the same procedure was performed as described in case 1. Thirty milliliters of tarry fluid were obtained from the right cyst, which was relevant to endometrioma, and $8 \mathrm{ml}$ of $99 \%$ ethanol were instilled and removed. The patient experienced one episode of severe lower abdominal pain after that. Immediately after the treatment, the size of the ovarian endometrioma as seen on ultrasound was unchanged. Cefdinir, $300 \mathrm{mg} /$ day orally, three times a day for three days, was given to prevent infection. The patient reported fever and headache on the 14th day posttreatment. Because increased levels of CRP, increased cyst size, and lower abdominal pain were detected on the 16th day posttreatment, piperacillin, $2.0 \mathrm{~g}$ intravenously, two times a day, as well as clarithromycin, $400 \mathrm{mg} / \mathrm{day}$ orally, two times a day, were administered. However, the patient continued to experience fever and abdominal pain, and a cyst with tenderness persisted despite antibiotic therapy, which indicated the presence of an ovarian abscess. Therefore, laparotomy was chosen for treatment. Enucleation of the right ovarian abscess 

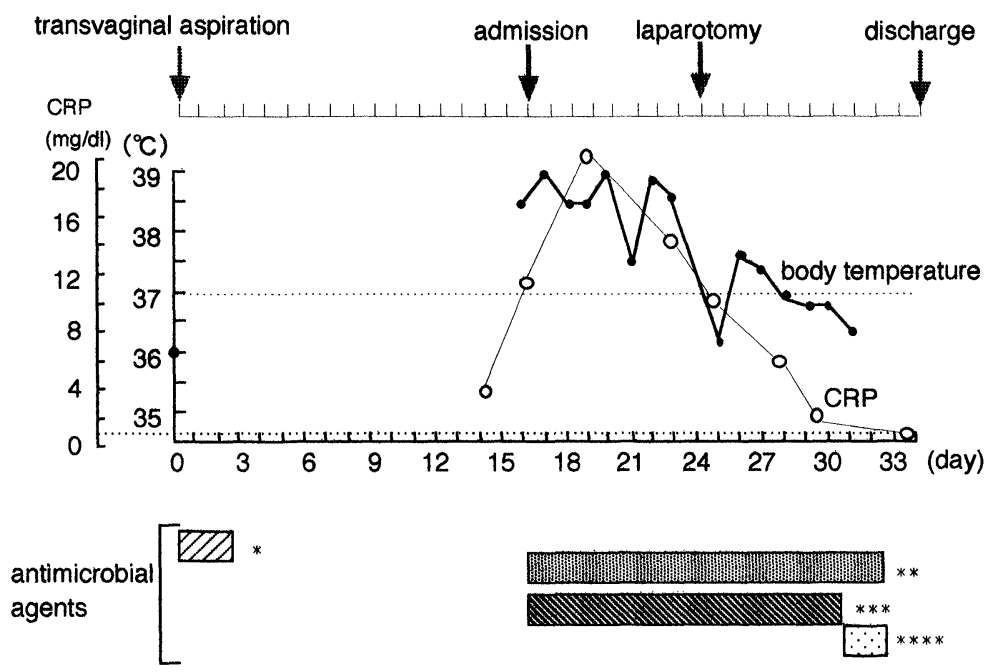

Fig. 2. Clinical course of case $2 . *=$ Cefdinir, $300 \mathrm{mg} /$ day orally, three times a day. $* *=$ Piperacillin, $2.0 \mathrm{~g}$ intravenously, two times a day. $* * *=$ Clarithromycin, $400 \mathrm{mg} /$ day orally, two times a day. $* * * *=$ Clindamycin, $1200 \mathrm{mg} /$ day intravenously, two times a day.

revealed purulent and malodorous fluid that showed $P$. magnus in culture, as well as on the results of a gram stain. Therefore, clindamycin sensitive for $P$. magnus was added from the 7 th postoperative day. After laparotomy, all symptoms disappeared (Fig. 2)

\section{DISCUSSION}

The use of ultrasound-guided aspiration for ovarian endometrioma has been clinically applied. ${ }^{3-5}$ This technique could obviate the need for surgical resection of cysts. ${ }^{3-5}$ We report two cases of ovarian abscess due to anaerobic bacterial infection after aspiration and ethanol fixation. With wide use of this technique, morbidity and mortality might be defined. , $^{, 7,8}$

We theorized about the cause of this infectious complication. P. magnus is an anaerobe present in normal intestinal and vaginal bacterial flora. The needle infected it into the ovarian endometrioma, even in the presence of ethanol. Alternatively, the anaerobic bacteria, being concealed in an avascular space of a chocolate cyst, could be activated due to the change of local host defense mechanism after ethanol tissue damage. We should screen for bacterial vaginosis before these procedures. Also, we should take care not to penetrate the intestine and the colon.
The clinical usefulness and overall safety of transvaginal ultrasound-guided aspiration and ethanol fixation of ovarian endometrioma has been evaluated, but in light of abscess complications, we might give additional special attention to the procedure.

\section{REFERENCES}

1. Barbieri RL, Ryan KJ: Danazol: endocrine pharmacology and therapeutic applications. Am J Obstet Gynecol 141:453-463, 1981.

2. Reich H, McGlynn F: Treatment of ovarian endometriomas using laparoscopic surgical techniques. J Reprod Med 31:577, 1986.

3. De Crespigny LC, Robinson HP, Davoren RAM, Fortune DW: Ultrasound-guided puncture for gynaecologic and pelvic lesions. Aust NZ J Obstet Gynaecol 25:227229, 1985 .

4. Diernaes E, Rasmussen J, Soerensen T, Hasch E: Ovarian cysts: management by puncture?. Lancet I:1084, 1987.

5. De Crespigny LC, Robinson HP, Davoren RAM, Fortune DW: The 'simple' ovarian cyst: aspirate or operate? Br J Obstet Gynaecol 96:1035-1039, 1989.

6. Berg JW, Robbins GE: A late look at the safety of aspiration biopsy. Cancer 15:826-827, 1962.

7. Padilla SL: Ovarian abscess following puncture of an endometrioma during ultrasound-guided oocyte retrieval. Hum Reprod 8:1282-1283, 1993.

8. Martino CR, Haaga JR, Bryan PJ: Secondary infection of an endometrioma following fine-needle aspiration. Radiology 151:53-54, 1984. 


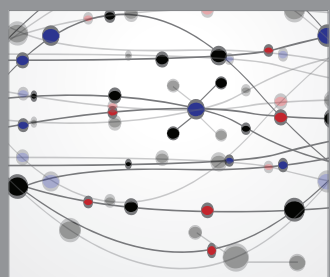

The Scientific World Journal
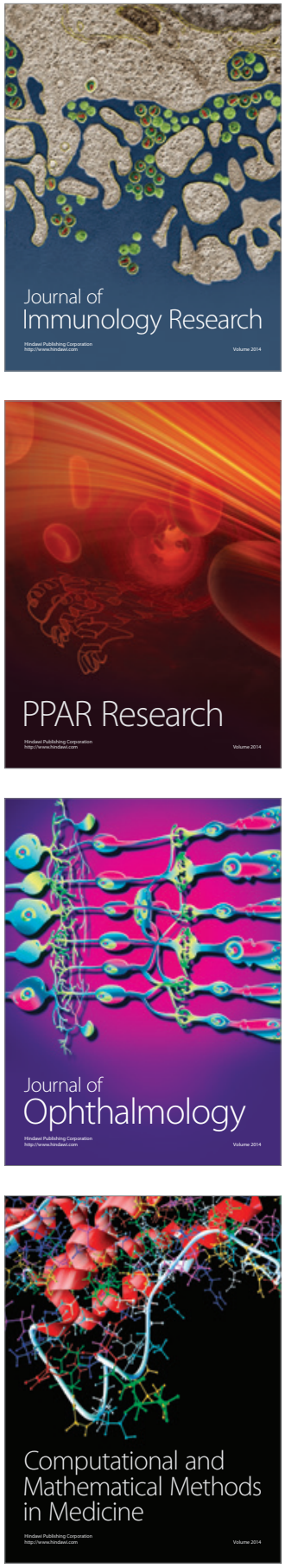

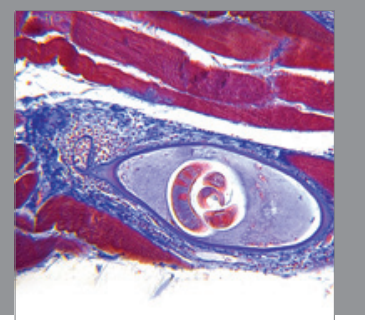

Gastroenterology

Research and Practice
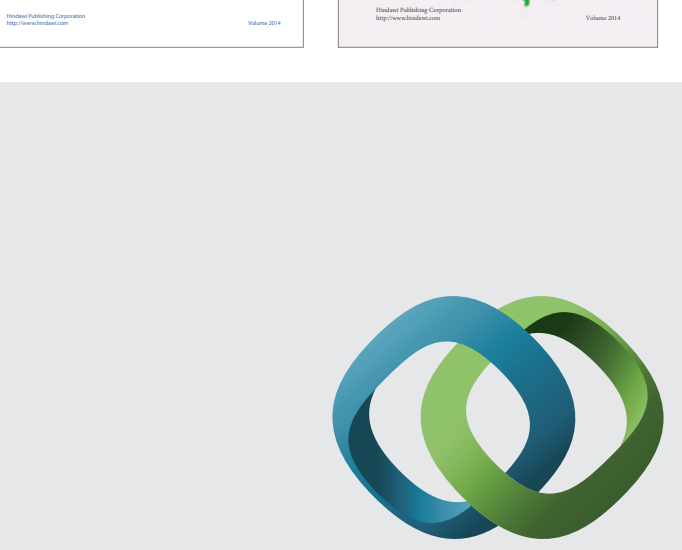

\section{Hindawi}

Submit your manuscripts at

http://www.hindawi.com
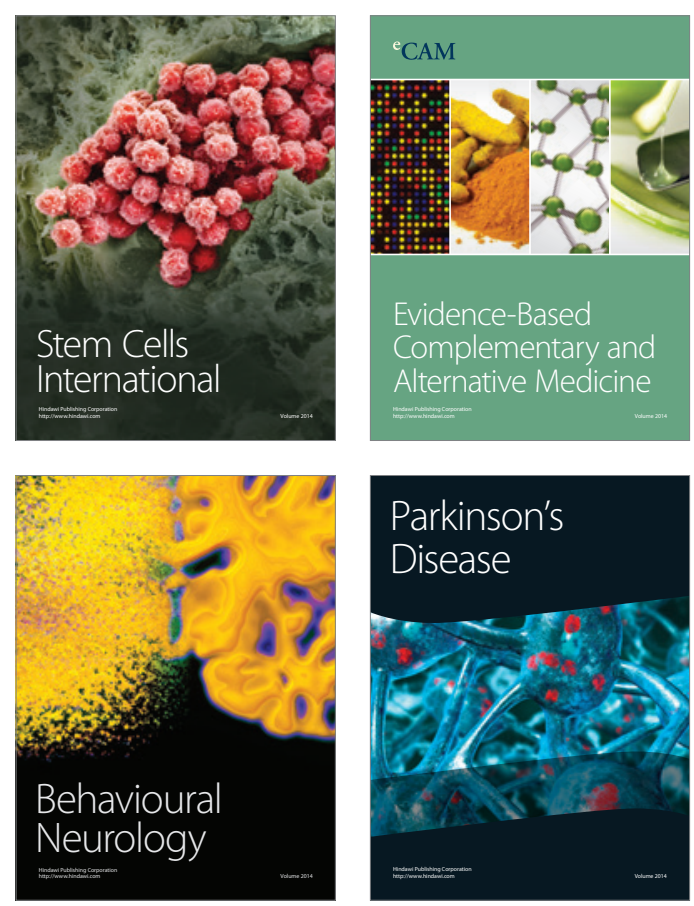

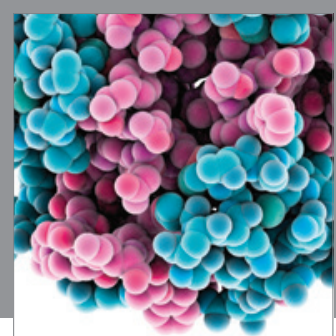

Journal of
Diabetes Research

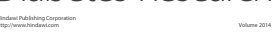

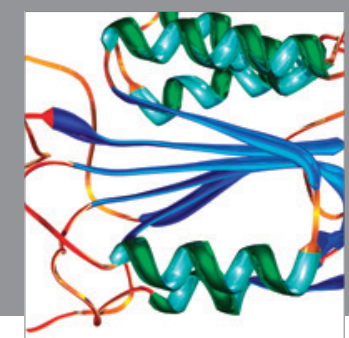

Disease Markers
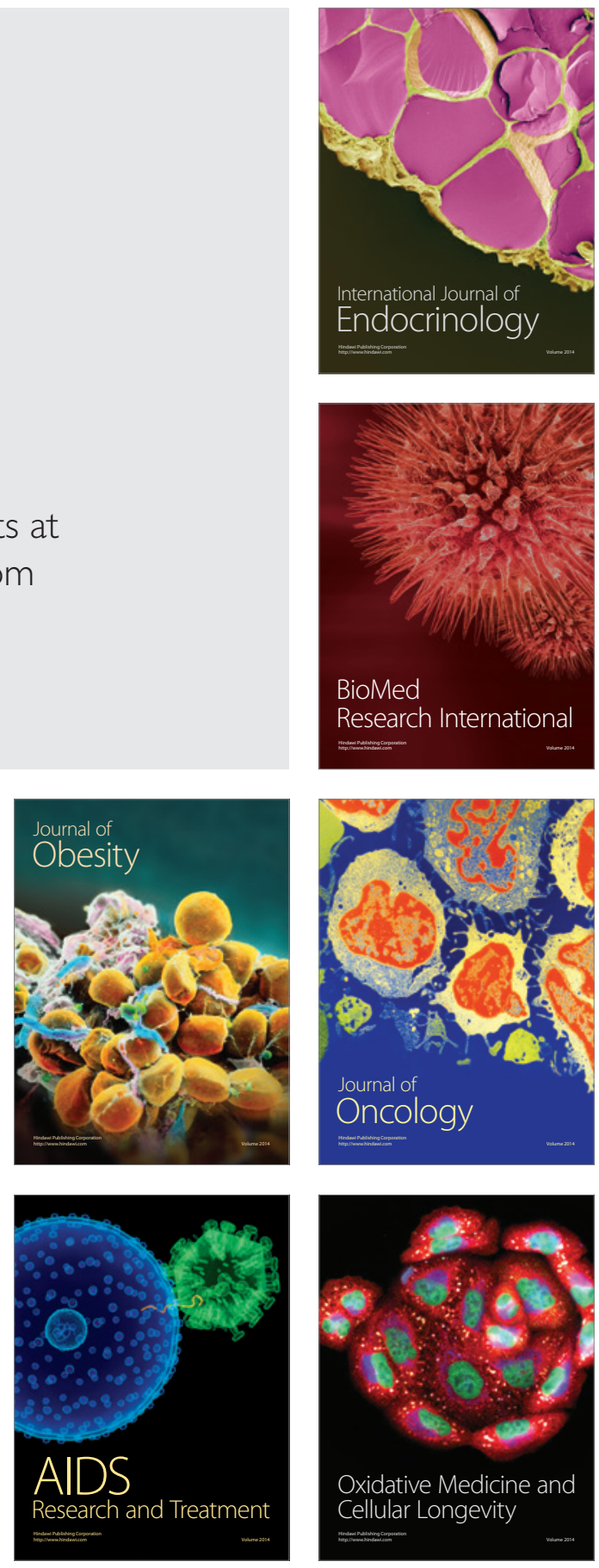